\title{
VARIAÇÃO SAZONAL DE MICRONUTRIENTES EM FOLHAS DE ACEROLEIRA (Malpighia emarginata DC.)
}

\section{Micronutrients seasonal variation in acerola leaves (Malpighia emarginata DC.)}

\author{
Rosiane de Lourdes Silva de Lima ${ }^{1}$, Dalmo Lopes de Siqueira ${ }^{2}$, Gilvan Barbosa Ferreira ${ }^{3}$, \\ Olmar Baller Weber, Jairo Osvaldo Cazetta ${ }^{5}$, Fernanda Fernandes de Melo Lopes ${ }^{6}$
}

\begin{abstract}
RESUMO
Os teores de micronutrientes nas folhas necessários para obter boa produtividade e qualidade de frutos de aceroleira (Malpighia emarginata DC.) e sua variação sazonal são pouco conhecidas. Para melhor entendimento da dinâmica de absorção de nutrientes e o desenvolvimento dessa frutífera avaliaram-se teores foliares de $\mathrm{Cu}, \mathrm{Fe}, \mathrm{Mn}$ e $\mathrm{Zn}$, em seis progênies de aceroleira no período de dezembro de 1999 a outubro de 2000. O estudo foi conduzido na Embrapa Agroindústria Tropical, em Pacajus, e envolveu 6 progênies (P52, P66, P78, P91, P93 e P97) e 6 épocas de amostragem das folhas (dezembro, fevereiro, abril, junho, agosto e outubro de 2000). A variação sazonal foi confirmada para os teores de $\mathrm{Cu}, \mathrm{Fe}, \mathrm{Mn}$ e $\mathrm{Zn}$ nas folhas, enquanto o $\mathrm{Zn}$ não sofreu alteração significativa nas progênies consideradas pela pesquisa. Os teores de $\mathrm{Cu}$ foram superiores em fevereiro, e os de $\mathrm{Fe}$ e $\mathrm{Mn}$ em agosto. As progênies apresentaram habilidade diferenciada na manutenção das concentrações de $\mathrm{Cu}, \mathrm{Mn}$ e $\mathrm{Zn}$ nas folhas. A melhor época para amostragem de folhas e diagnóstico do estado nutricional foi em outubro, início do florescimento das aceroleiras, quando os teores de $\mathrm{Cu}, \mathrm{Fe}, \mathrm{Mn}$ e Zn devem ser superiores a 3,125, 80 e $15 \mathrm{mg} / \mathrm{kg}$.
\end{abstract}

Termos para indexação: Malpighia emarginata, sazonalidade e nutrição vegetal.

\section{ABSTRACT}

Micronutrients seasonal variation in leaves that are necessary to get good productivity and quality in (Malpighia emarginata DC.) (Acerola) fruits are little known. For better acquaintance of the dynamics or absorption and the of this plant, foliar amount of $\mathrm{Cu}, \mathrm{Fe}, \mathrm{Mn}$ and $\mathrm{Zn}$ was evaluated in six lineages of acerola plant from December,1999 to October,2000. Field experiments were carried out at the Embrapa Tropical Agroindustry, Pacajus experimental field, with 6 lineages (P52, P66, P78, P91, P93 and P97) and 6-leaf-sampling (December, February, April, June, August and October of 2000). The seasonal variation was confirmed in leaves for amounts of $\mathrm{Cu}, \mathrm{Fe}, \mathrm{Mn}$ and $\mathrm{Zn}$. The amount of $\mathrm{Zn}$ did not suffer significant alteration in the lineages considered for the research. The amount of $\mathrm{Cu}$ was higher in February, while $\mathrm{Fe}$ and $\mathrm{Mn}$ in August. The lineages presented different abilities to keep $\mathrm{Cu}, \mathrm{Mn}$ and $\mathrm{Zn}$ concentrations in leaves. For diagnosis of the plant nutritional state, the best time for leaf sampling was at the beginning of the bloom, during October, when the amount of $\mathrm{Cu}, \mathrm{Fe}, \mathrm{Mn}$ and $\mathrm{Zn}$ reached the contends of than 3, 125, 80 and $15 \mathrm{mg} / \mathrm{kg}$.

Index terms: Malpighia emarginata, sazonality and vegetal nutrition.

(Recebido em 12 de dezembro de 2006 e aprovado em 10 de setembro de 2007)

\section{INTRODUÇÃO}

A aceroleira (Malpighia emarginata DC.) é uma planta de crescimento intenso e que apresenta alta demanda por nutrientes, quando cultivada em condições de clima e manejo adequados (LIMA et al., 2006). As plantas iniciam a produção, já a partir do primeiro ano de cultivo, ocasião em que podem produzir até $2 \mathrm{~kg}$ de frutos frescos. Aos seis anos produz, em média, $47 \mathrm{~kg}$ cada planta (BRAGA
SOBRINHO et al., 2001). Entretanto, há registros de produção de até $179,2 \mathrm{~kg}$ planta ano $^{-1}$ (GONZAGA NETO et al., 1999). Apesar da produtividade média no Brasil ser baixa, (cerca de $10 \mathrm{t} \mathrm{ha}^{-1}$ ), diversos acessos podem produzir acima de 40 t ha ano $^{-1}$, quando irrigada, adubada e manejada adequadamente (OLIVEIRA \& SOARES FILHO, 1999).

Segundo Andrade (2004), a determinação de nutrientes que são exportados pelos frutos é importante para fazer cálculos de reposição com adubos de modo a

\footnotetext{
1Engenheira Agrônoma, Doutora em Agronomia - Produção Vegetal - Embrapa Algodão - Rua Osvaldo Cruz, 1123 - Centenário - Cx. P. 174 - $58107-720$ Campina Grande, PB - limarosiane@yahoo.com.br

${ }^{2}$ Doutor, Professor Adjunto - Departamento de Fitotecnia - Universidade Federal de Viçosa/UFV - Campus Universitário - Avenida Peter Henry Rolfs, s/n - 36570-000 - Viçosa, MG - siqueira@ufv.br

${ }^{3}$ Engenheiro Agrônomo, Doutor em Solos e Nutrição de Plantas - Pesquisa e Desenvolvimento - Embrapa Roraima - Rodovia Br 174 , Km 08 - Distrito Industrial - 69301-970 - Boa Vista, RR - gilvan@cpafrr.embrapa.br

${ }^{4}$ EngenheiroAgrônomo, Doutor em Agronomia/Ciência do Solo - Embrapa Agroindústria Tropical - Rua Doutora Sara Mesquita, 2270 - Pici - 60511-110 Fortaleza, CE - weber@cnpat.embrapa.br

${ }^{5}$ Doutor, Professor Adjunto - Departamento de Tecnologia - Universidade Estadual Paulista/UNESP - Via de Acesso Professor Paulo Donato Castellane s/n - 14884-900 - Jaboticabal, SP - cazetta@fcav.unesp.br

${ }^{6}$ Engenheira Agrícola, Doutoranda em Engenharia de Processos - Universidade Federal de Campina Grande/UFCG - Rua Janúncio Ferreira, 353 Centro - 58102-555 - Campina Grande, PB - fndlopes@gmail.com
} 
garantir frutos de qualidade. Com esse procedimento, é possível manter equilibrada a fertilidade do solo e garantir produtividade e a rentabilidade da cultura. Além disso, o conhecimento dos teores de nutrientes nas folhas é uma medida efetiva para acompanhar a evolução do estado nutricional da cultura e para organizar estratégias eficientes de manejo da adubação mineral visando altas produtividades (LIMA et al., 2006). Villela \& Lacerda (1992) mencionaram que a variação sazonal na concentração dos elementos minerais nas folhas está fortemente relacionada aos mecanismos de absorção e de retranslocação. Segundo Marschner et al. (1996), existem diferenças entre as espécies vegetais e mesmo entre variedades pertencentes a uma mesma espécie, quanto à eficiência na utilização dos nutrientes para a produção de compostos orgânicos, dependendo da sua disponibilidade no solo.

O conhecimento da variação dos teores de nutrientes nas folhas permite inferir sobre as exigências metabólicas da planta, fornecendo base para o entendimento dessas variações e suas implicações sobre o comportamento da planta no ecossistema (MARSCHNER et al., 1996).

O monitoramento da variação sazonal de teores foliares permite estabelecer padrões para o manejo nutricional das plantas visando o alcance e manutenção de alta produtividade e qualidade do frutos de acerola.

Objetivou-se, neste trabalho avaliar a variação sazonal dos teores de $\mathrm{Cu}, \mathrm{Fe}, \mathrm{Mn}$ e $\mathrm{Zn}$ em folhas em uma coleção de 6 progênies de aceroleira, no período de dezembro de 1999 a outubro de 2000.

\section{MATERIAL E MÉTODOS}

O ensaio foi conduzido na coleção de progênies de aceroleira da Embrapa Agroindústria Tropical, em Pacajus, CE, no período de dezembro de 1999 a outubro de 2000.

No experimento, adotou-se o esquema de parcelas subdivididas, sendo as parcelas constituídas por 6 progênies (P-52, P-66, P-78, P-91, P-93 e P-97) e as subparcelas por 6 épocas de avaliação (dezembro de 1999, fevereiro, abril, junho, agosto e outubro de 2000). O delineamento foi de blocos casualizados, com 4 repetições, e uma planta para cada unidade experimental.

As características do solo foram determinadas no laboratório de Solos e Água da Embrapa, podendo ser observadas na Tabela 1.

Entre os meses de dezembro de 1999 e outubro de 2000 foram registrados $1122,2 \mathrm{~mm}$ de chuva, sendo que nos meses de janeiro a maio de 1999 choveu $845 \mathrm{~mm}$. O menor volume de chuva foi registrado nos meses de setembro e outubro de 2000 (36,1 e 2,0 mm). A quantidade de chuva foi considerada satisfatória para o pleno desenvolvimento da cultura.

As plantas foram cultivadas em regime de sequeiro, utilizando-se o espaçamento $4 \mathrm{~m} \times 3 \mathrm{~m}$. A fertilização das plantas foi realizada todos os meses, no período de dez/ 1999 a out/2000, aplicando-se em sulcos rasos abertos em

Tabela 1 - Características químicas do solo em duas camadas do pomar de aceroleira, no campo experimental de Pacajus, Pacajus, CE.

\begin{tabular}{|c|c|c|}
\hline \multirow[b]{2}{*}{ Características } & \multicolumn{2}{|c|}{ Profundidade do Solo } \\
\hline & 0 a $0,20 \mathrm{~m}$ & 0,20 a $0,40 \mathrm{~m}$ \\
\hline $\mathrm{pH} \mathrm{CaCl}_{2}{ }^{(1)}$ & 4,8 & 4,3 \\
\hline $\mathrm{P}\left(\mathrm{mg} / \mathrm{dm}^{3}\right)^{(2)}$ & 27,7 & 6,4 \\
\hline $\mathrm{K}^{+}\left(\mathrm{mmol}_{\mathrm{c}} / \mathrm{dm}^{3}\right)^{(2)}$ & 1,4 & 1,5 \\
\hline $\mathrm{Ca}^{2+}\left(\mathrm{mmol}_{\mathrm{c}} / \mathrm{dm}^{3}\right)^{(3)}$ & 16,5 & 5,5 \\
\hline $\mathrm{Mg}^{2+}\left(\mathrm{mmol}_{\mathrm{c}} / \mathrm{dm}^{3}\right)^{(3)}$ & 6,8 & 2,5 \\
\hline $\mathrm{Al}^{3+}\left(\mathrm{mmol}_{\mathrm{c}} / \mathrm{dm}^{3}\right)^{(3)}$ & 0,15 & 0,20 \\
\hline $\mathrm{H}+\mathrm{Al}\left(\mathrm{mmol}_{\mathrm{c}} / \mathrm{dm}^{3}\right)^{(4)}$ & 1,3 & 1,5 \\
\hline $\mathrm{SB}\left(\mathrm{mmol}_{\mathrm{c}} / \mathrm{dm}^{3}\right)$ & 29,3 & 13,4 \\
\hline CTC Total $\left(\mathrm{mmol}_{\mathrm{c}} / \mathrm{dm}^{3}\right)$ & 30,8 & 15,1 \\
\hline $\mathrm{V}(\%)$ & 95 & 89 \\
\hline
\end{tabular}

\footnotetext{
(1) $\mathrm{pH} \mathrm{em} \mathrm{CaCl} 2$ 0,01 mol/L; ${ }^{(2)}$ Mehlich - 1; ${ }^{(3)} \mathrm{KCl} 1 \mathrm{~mol} / \mathrm{L} ;{ }^{(4)}$ Acetato de cálcio 0,5 mol/L (EMBRAPA, 1997).
} 
meia-lua na projeção da copa, $30 \mathrm{~g}$ de uréia, $50 \mathrm{~g}$ fosfato monoamônico (MAP), $20 \mathrm{~g}$ de sulfato de magnésio e $40 \mathrm{~g}$ de cloreto de potássio. Cada planta recebeu ainda a partir de janeiro de 2000 a cada 60 dias, 90 g de uréia, 72 g de cloreto de potássio e $20 \mathrm{~g}$ de sulfato de magnésio.

A amostragem de folhas foi realizada a cada 60 dias, ocorrendo a primeira em dezembro de 1999 e a última em outubro de 2000, sendo coletadas de quatro plantas/ progênie, das 6 progênies (P-52, P-66, P-78, P-91, P-93 e P97) selecionadas. As amostras foram coletadas nos terços medianos dos ramos sem frutos da porção superior da copa de quatro plantas das progênies, sendo posteriormente lavadas em água deionizada, acondicionadas em sacos de papel e secas em estufa, com circulação forçada de ar, a $70^{\circ} \mathrm{C}$, até peso constante. $\mathrm{O}$ material seco foi passado em moinho tipo Willey, com peneira de 20 mesh, e as amostras submetidas à digestão em solução nítrico-perclórica. Em seguida, dosaram-se os teores de $\mathrm{Cu}, \mathrm{Fe}, \mathrm{Mn}$ e $\mathrm{Zn}$ por espectrofotometria de absorção atômica.

Os dados coletados foram submetidos à análise de variância pelo teste $\mathrm{F}$ e pelo teste de múltipla comparação de médias (Tukey), ao nível de $5 \%$ de probabilidade.

\section{RESULTADOS E DISCUSSÃO}

Detectaram-se efeitos significativos (5\% pelo Teste F) dos tratamentos (progênies e épocas do ano) sobre os teores de micronutrientes estudados, observando-se que houve interação significativa entre progênie e época para o $\mathrm{Cu}$, efeitos isolados de época para o $\mathrm{Fe}$, efeito isolado de progênie e de época para o Mn e efeito de época para o Zn, respectivamente (Tabela 2 ).
Pela Tabela 3, verifica-se predominância de altos teores de $\mathrm{Cu}$, na amostragem de fevereiro $\left(7,6 \mathrm{mg} \mathrm{kg}^{-1}\right)$, em comparação ao menor teor do elemento detectado em outubro de 2000 (2,4 $\left.\mathrm{mg} \mathrm{kg}^{-1}\right)$. Salienta-se, de acordo com a fenologia da planta, que nessas épocas havia alta incidência de frutos e flores. Além disso, pode-se acrescentar que, por se tratar de uma planta que apresenta surtos de crescimento vegetativo, floração e frutificação, concomitantemente, fica difícil associar a concentração do nutriente com a fenologia da planta.

Na progênie P-97, observou-se maior tendência em concentrar $\mathrm{Cu}$ em tecidos foliares, quando comparada às demais. A reserva de $\mathrm{Cu}$, disponível no solo, é a matéria orgânica (FERREIRA, 2003) e sua liberação depende da abundância relativa e da sua mineralização. A atividade microbiana afeta a mineralização e é restringida quando há redução da umidade do solo, o que ocorre naturalmente com o fim do período chuvoso; por problemas no sistema de irrigação, usado na área experimental, não foi possível evitar a ocorrência de estresse hídrico no período seco. Esse fato, associado à baixa reserva do solo arenoso e à aplicação de adubos, que contêm cátions que competem pela absorção, nos sítios das membranas celulares das células das raízes $\left(\mathrm{Fe}^{2+}, \mathrm{Mn}^{2+}, \mathrm{Zn}^{2+}, \mathrm{Mg}^{2+}\right.$ e $\left.\mathrm{K}^{+}\right)$, podem explicar, em parte, a redução dos teores de $\mathrm{Cu}$, observada entre agosto e outubro de 2000. Assim, pode-se supor uma lenta acumulação foliar nos meses seguintes, com o início da estação chuvosa, até atingir o máximo em fevereiro de 2000, caindo, posteriormente, por efeito de diluição e competição pelo nutriente com outros órgãos da planta (folhas novas, flores e frutos). Os teores de $\mathrm{Cu}$ estiveram abaixo da faixa do nível proposto por Oliveira (2004), que prevê variações de 5 a $15 \mathrm{mg} \mathrm{kg}^{-1}$.

Tabela 2 - Resumo da análise de variância para teores de Cu, Fe, Mn e Zn, nas folhas de aceroleira em função da progênie e da época de avaliação. Pacajus, CE, 1999/2000.

\begin{tabular}{lccccc}
\hline \multirow{2}{*}{ Fontes da variação } & G.L. & \multicolumn{4}{c}{ Quadrados médios } \\
\cline { 3 - 6 } & & $\mathrm{Cu}$ & $\mathrm{Fe}$ & $\mathrm{Mn}$ & $\mathrm{Zn}$ \\
\hline Bloco & 3 & 0,55 & 203,6 & 2704,8 & 24,9 \\
Progênie & 5 & 9,94 & 2923,6 & $12841,9^{*}$ & 110,5 \\
Erro (A) & 15 & 6,94 & 1622,6 & 1423,7 & 115,6 \\
Época & 5 & $16,14^{*}$ & $33835,5^{*}$ & $8001,7^{*}$ & $66,4^{*}$ \\
P X E & 25 & $2,88^{*}$ & 1424,2 & 1649,4 & 30,6 \\
Erro (B) & 90 & 1,03 & 1385,9 & 1082,6 & 27,5 \\
\hline CVa (\%) & - & 56,4 & 36,9 & 30,8 & 53,8 \\
\hline CVb (\%) & - & 21,7 & 34,1 & 26,9 & 26,2 \\
\hline
\end{tabular}

*significativo pelo teste $\mathrm{F}$, a $5 \%$ de probabilidade. 
LIMA, R. de L. S. de et al.

Tabela 3 - Teores médios de Cu e Mn ( $\left.\mathrm{mg} \mathrm{kg}^{-1}\right)$, na massa seca foliar de progênies de aceroleira. Pacajus, CE, $1999 / 2000$.

\begin{tabular}{|c|c|c|c|c|c|c|c|}
\hline \multirow{3}{*}{ Genótipos } & \multicolumn{6}{|c|}{$\mathrm{Cu}$} & \multirow{3}{*}{$\begin{array}{c}\text { Mn } \\
\text { Média de } 6 \\
\text { épocas }\end{array}$} \\
\hline & \multicolumn{6}{|c|}{ Épocas de amostragem } & \\
\hline & $\mathrm{dez} / 1999$ & fev/2000 & abr/2000 & jun/2000 & $\mathrm{ago} / 2000$ & out $/ 2000$ & \\
\hline P 52 & B $4,2 \mathrm{a}$ & A $6,5 \mathrm{ab}$ & B $3,7 \mathrm{a}$ & B 3,9a & B $2,8 \mathrm{a}$ & B $2,4 b$ & $099,8 \mathrm{bc}$ \\
\hline P 66 & $\mathrm{AB} 5,6 \mathrm{a}$ & A 7,6a & $\mathrm{AB} 5,5 \mathrm{a}$ & $\mathrm{AB} 5,9 \mathrm{a}$ & $\mathrm{B} 4,0 \mathrm{a}$ & B 4,0ab & $152,3 \mathrm{a}$ \\
\hline P 78 & BC 3,6a & A $7,1 \mathrm{a}$ & $\mathrm{BC} 4,5 \mathrm{a}$ & AB 5,3a & BC $3,5 \mathrm{a}$ & $\mathrm{C} 2,6 \mathrm{~b}$ & $134,9 \mathrm{ab}$ \\
\hline P 91 & A $4,2 \mathrm{a}$ & A $4,0 \mathrm{~b}$ & A $4,7 \mathrm{a}$ & A $4,7 \mathrm{a}$ & A $4,5 \mathrm{a}$ & A $3,0 b$ & $128,1 \mathrm{ab}$ \\
\hline P 93 & A $4,7 \mathrm{a}$ & A $5,4 \mathrm{ab}$ & A $5,1 \mathrm{a}$ & A $4,1 \mathrm{a}$ & A $4,8 \mathrm{a}$ & A $3,5 \mathrm{ab}$ & $129,5 \mathrm{ab}$ \\
\hline P 97 & A $5,6 \mathrm{a}$ & A $5,8 \mathrm{a}$ & A $5,1 \mathrm{a}$ & A $4,6 a$ & A $5,8 \mathrm{a}$ & A $6,0 \mathrm{a}$ & $090,3 \mathrm{c}$ \\
\hline
\end{tabular}

Médias seguidas das mesmas letras, maiúscula na linha entre as épocas de amostragem e minúscula na coluna, não diferem entre si pelo teste de Tukey $(\mathrm{p}<0,05)$.

Neste trabalho, verificaram-se teores de $\mathrm{Cu}$ tão baixos quanto $2,4 \mathrm{mg} \mathrm{kg}^{-1}$ (outubro de 2000) para as progênies sem que essas apresentassem sintomas visuais de deficiência nutricional. Provavelmente, havia uma carência do elemento no solo, considerando que esse solo tem baixo teor de matéria orgânica $\left(11,48 \mathrm{~g} \mathrm{~kg}^{-1}\right)$ e a adubação empregada tinha cátions competidores, a exemplo de $\mathrm{Fe}^{2+}, \mathrm{Mn}^{2+}, \mathrm{Zn}^{2+}, \mathrm{Mg}^{2+}$, entre outros. Isso, provavelmente, diminuiu a absorção e a acumulação do $\mathrm{Cu}$ nas folhas analisadas.

Observou-se efeito significativo pelo teste F, para os teores de Fe dentro das épocas estudadas (Tabela 2). Os teores de Fe foram baixos nas amostragens de fevereiro, abril e junho (Tabela 4), comparados com o limite inferior das faixas apresentadas por Amaral et al. (2002), de 53 a $118 \mathrm{mg} \mathrm{kg}^{-1}$, e por Oliveira (2004), de 50 a $100 \mathrm{mg} \mathrm{kg}^{-1}$. Os maiores teores do elemento foram detectados em agosto de $2000\left(202,87 \mathrm{mg} \mathrm{kg}^{-1}\right)$, época caracterizada pela presença de flores em todas as progênies estudadas. Destaque-se que esse teor encontra-se na faixa diagnosticada por Amaral et al. (2002) e Oliveira (2004).

A intensa oscilação nos teores de Fe encontrados, ora se situando em altas concentrações, ora se apresentando na faixa de suficiência, reflete a dinâmica de Fe no tecido foliar da aceroleira, tendendo a concentrar-se no tecido com o aumento da idade. Também reflete o desenvolvimento fenológico da cultura da acerola, considerando os dados pluviométricos da localidade. Houve uma precipitação mensal superior a $100 \mathrm{~mm}$, a partir de janeiro de 2000. Até então as plantas estavam com folhas velhas que suportaram o período seco anterior. Portanto, tecidos velhos enriquecidos de Fe que têm baixa mobilidade no floema, o que é concordante com as observações feitas por Marschner et al. (1996). Com o início do período chuvoso ocorreu crescimento de novos ramos e folhas, associado com o desenvolvimento e crescimento de órgãos reprodutivos. Esses novos tecidos têm, pois, motivos para apresentarem baixos teores de Fe: são novos, não sofreram acúmulo de Fe e estão em forte expansão; e competem com os frutos pelo Fe absorvido pelas raízes e translocados no xilema. Isso leva a sugerir suprimento preferencial de $\mathrm{Fe}$ para os frutos entre os meses de abril e junho, nos períodos de forte demanda do nutriente pelos frutos e pelos ramos vegetativos.

Nas amostragens de abril e junho/2000, os teores de Fe foram abaixo do valor médio de $80,6 \mathrm{mg} \mathrm{kg}^{-1}$ diagnosticado por Amaral et al. (2002). Entretanto, sintomas de deficiência não foram verificados nesse período nas diferentes progênies. Marinho et al. (2002) relataram deficiências de Fe em solos alcalinos, arenosos ou calagens excessivas. Comportamento não observado nesse trabalho para aceroleira.

Os teores de Mn variaram em razão das progênies (Tabela 2) e épocas de amostragem das folhas (Tabela 4). Maiores teores médios foram detectados em agosto de $2000\left(145,42 \mathrm{mg} \mathrm{kg}^{-1}\right)$ e os menores em fevereiro do mesmo ano agrícola $\left(98,25 \mathrm{mg} \mathrm{kg}^{-1}\right)$, para as progênies avaliadas. Os teores observados encontram-se acima da faixa considerada por Oliveira (2004), 15-50 mg kg-1 . Os altos valores detectados nesse estudo podem explicar, em parte, os baixos teores de $\mathrm{Cu}$ nas folhas (Tabela 3 ). De acordo com Marschner et al. (1996), esses nutrientes competem entre si na absorção celular e o excesso de um reduz a concentração dos demais nutrientes. A alta quantidade de nitrogênio aplicada pode ter ajudado na elevação dos teores de Mn, como constatado por Marinho et al. (2002) nos mamoeiros 'Solo' e 'Formosa'. Além disso, a acidez do solo usado pode favorecer uma maior absorção de Mn, como discutido por Malavolta et al. (1997) e Marschner et al. (1996). 
Tabela 4 - Teores médios de Fe, $\mathrm{Zn} \mathrm{e} \mathrm{Mn}\left(\mathrm{mg} \mathrm{kg}^{-1}\right)$, na massa seca foliar de genótipos de aceroleira, em seis épocas de amostragem. Pacajus, CE, 1999/2000.

\begin{tabular}{lccc}
\hline \multirow{2}{*}{ Época de amostragem } & \multicolumn{3}{c}{ Nutrientes $\left(\mathrm{mg} \mathrm{kg}^{-1}\right)$} \\
\cline { 2 - 4 } & $\mathrm{Fe}$ & $\mathrm{Zn}$ & $\mathrm{Mn}$ \\
\hline Dezembro/1999 & $145,65 \mathrm{a}$ & $20,61 \mathrm{ab}$ & $103,22 \mathrm{bc}$ \\
Fevereiro/2000 & $90,40 \mathrm{~b}$ & $18,73 \mathrm{ab}$ & $98,25 \mathrm{c}$ \\
Abril/2000 & $69,24 \mathrm{~b}$ & $17,50 \mathrm{~b}$ & $126,85 \mathrm{ab}$ \\
Junho/2000 & $69,17 \mathrm{~b}$ & $20,19 \mathrm{ab}$ & $134,63 \mathrm{a}$ \\
Agosto/2000 & $129,41 \mathrm{a}$ & $22,30 \mathrm{a}$ & $145,42 \mathrm{a}$ \\
Outubro/2000 & $151,15 \mathrm{a}$ & $20,57 \mathrm{ab}$ & $126,52 \mathrm{ab}$ \\
\hline
\end{tabular}

Médias seguidas das mesmas letras minúscula na coluna, não diferem entre si pelo teste de Tukey, $(\mathrm{p}<0,05)$.

Os teores de Mn oscilaram nas amostragens de dezembro de 1999 e fevereiro de 2000 (Tabela 4), de forma semelhante ao observado para o $\mathrm{Fe}$, há tendência de acúmulo do Mn no tecido foliar ao final do período chuvoso. Provavelmente, maior acidificação do solo pelas adubações efetuadas no período anterior (período agrícola de 1999) afeta a presença de sítios de redução do potencial de redução do elemento, aumentando a concentração de Mn nos tecidos das folhas mais velhas.

Os teores de $\mathrm{Zn}$ mantiveram-se entre $17,5 \mathrm{mg} \mathrm{kg}^{-1}$ a 22,3 $\mathrm{mg} \mathrm{kg}^{-1}$ (Tabela 4). Os valores acima desse intervalo estão na faixa adequada de acordo com Jones Júnior et al. (1991), enquanto os demais se apresentaram em baixos níveis, em comparação com os níveis de concentração propostos por esses autores.

Os teores médios de $\mathrm{Zn}$ observados encontram-se dentro da faixa observada por Amaral et al. (2002) que é de 16,9 a $24,1 \mathrm{mg} \mathrm{kg}^{-1}$ de $\mathrm{Zn}$, medidos na porção mediana da copa das plantas. $\mathrm{O}$ limite superior encontra-se abaixo do nível observado (29,8 $\left.\mathrm{mg} \mathrm{kg}^{-1}\right)$ pelo autor. Por sua vez, Oliveira (2004) considerou o teor de 30 a $50 \mathrm{mg} \mathrm{kg}^{-1}$, adequado para aceroleira. Destaque-se que, no presente trabalho, não se observaram sintomas característicos de deficiência do elemento. Isso mostra que a faixa de concentração do Zn é imprecisa, podendo estar mais relacionada à diferença varietal do que ao período de amostragem. As partes da copa e do ramo amostradas também podem estar influindo na definição dos valores adequados e precisam de mais estudos. O comportamento desse elemento foi semelhante ao observado para o Fe, embora com variações menos intensas nas épocas de amostragem (Tabela 4).

De acordo com Amaral et al. (2002) as amostragens de folhas para diagnosticar o estádio nutricional de plantas devem ser repetidas em períodos que sucedem as colheitas, pois elas podem esgotar as reservas nutricionais da planta.
Como a aceroleira, em condições tropicais, apresenta fluxo contínuo de produção e é influenciada pelos tratos culturais, fica difícil padronizar a época das grandes colheitas. A interpretação dos teores observados deve sugerir que, para cada nutriente, existe um período mais crítico influenciado por fatores climáticos e pela progênie. Por praticidade, no entanto, nas amostras de tecidos foliares, colhidas no início do florescimento, no terço-médio dos ramos e na porção superior da copa, os teores de $\mathrm{Cu}, \mathrm{Fe}, \mathrm{Mn}$ e $\mathrm{Zn}$ devem ser superiores a $3,125,80$ e $15 \mathrm{mg} \mathrm{kg}^{-1}$, respectivamente, para garantir um bom estado nutricional e produtividade elevada para a maioria das progênies testadas.

\section{CONCLUSÕES}

Houve variação sazonal de $\mathrm{Cu}$, Fe e $\mathrm{Mn}$ e $\mathrm{Zn}$ nas folhas de aceroleira, sendo constatados os maiores teores de $\mathrm{Fe}$ e $\mathrm{Mn}$ em agosto e de $\mathrm{Cu}$ em fevereiro.

As concentrações de $\mathrm{Zn}$ nas folhas de aceroleira não foram influenciadas nas progênies consideradas pela pesquisa.

As progênies de aceroleira têm habilidades diferenciadas na manutenção dos teores de $\mathrm{Cu}, \mathrm{Mn}$ e $\mathrm{Zn}$ nas folhas.

A amostragem de folhas para diagnosticar o estado nutricional da aceroleira deve ser feita em outubro, início do florescimento, quando os teores de $\mathrm{Cu}, \mathrm{Fe}, \mathrm{Mn}$ e $\mathrm{Zn}$ são superiores a 3, 125, 80 e $15 \mathrm{mg} \mathrm{kg}^{-1}$, respectivamente, na maioria das progênies de acerola estudadas.

\section{REFERÊNCIAS BIBLIOGRÁFICAS}

AMARAL, J. F. T. do; BRUCKNER, C. H.; MARTINEZ, H. E. P.; CRUZ, C. D.; GODOY, C. L.; CAIXETA, S. L. Determination of leaf sampling techniques to assess the nutritional status of Barbados cherry (Malpighia emarginata D.C.). Fruits, [S.1.], v. 57, p. 161-171, 2002. 
ANDRADE, L. R. M. Corretivos e fertilizantes para culturas perenes e semiperenes. In: SOUSA, D. M. G.; LOBATO, R (Eds.). Cerrado: correção do solo e adubação. 2. ed. Brasília, DF: Embrapa Informação Tecnológica, 2004. p. 317-366.

BRAGA SOBRINHO, R.; BANDEIRA, C. T.; ALVES, R. E. Acerola: a cereja tropical. Fortaleza: Embrapa Agroindústria Tropical, 2001.

EMPRESA BRASILEIRA DE PESQUISA AGROPECUÁRIA. Manual de métodos de análises de solo. 2. ed. Rio de Janeiro: Ministério da Agricultura e do Abastecimento, 1997. 212 p.

FERREIRA, G. B. Dinâmica das frações de micronutrientes catiônicos e esgotamento de formas disponíveis de boro, cobre, ferro, manganês e zinco em Solos de Minas Gerais. 2003. 169 f. Tese (Doutorado) - Universidade Federal de Viçosa, Viçosa, 2003.

GONZAGA NETO, L.; MATTUZ, B. H.; SANTOS, C. A. F. Caracterização agronômica de clones de aceroleira (Malpighia spp) na região do submédio São Francisco. Revista Brasileira de Fruticultura, Jaboticabal, v. 21, n. 2, p. 110-115, 1999.

JONES JUNIOR, J. B.; WOLF, B.; MILL, H. A. Plant analysis handbook, a pratical samplig, preparation, analysis, and interpretation guide. Anthens: Micro-Macro, 1991.

LIMA, R. L. S.; SIQUEIRA, D. L.; WEBER, O. B.; CECON, P. R. Teores de maconutrientes em mudas de aceroleira (Malpighia emarginata D.C.) em função da composição do substrato. Ciência e Agrotecnologia, Lavras, v. 30, n. 6, p. 1110-1115, 2006.
MALAVOLTA, E.; VITTI, G. C.; OLIVEIRA, S. A. de. Avaliação do estado nutricional das plantas: princípios e aplicações. 2. ed. Piracicaba: Potafos, 1997. 319 p.

MARINHO, C. S.; MONNERAT, P. H.; CARVALHO JÚNIOR, A. C. de; MARTINS, S. L. D.; VIEIRA, A. Análise química do pecíolo e limbo foliar como indicadora do estado nutricional dos mamoeiros 'Solo' e 'Formosa'. Scientia Agrícola, Piracicaba, v. 59, n. 2, p. 373-381, 2002.

MARSCHNER, H.; KIRKBY, E. A.; CAKMAK, I. Effect of mineral nutritional status on shoot-root partitioning of photoassimilates and cycling of mineral nutrients. Journal of Experimental Botany, Oxford, v. 47, p. 1255-1263, 1996.

OLIVEIRA, J. R. P. de; SOARES FILHO, W. dos S. Situação da cultura da acerola no Brasil e ações da Embrapa Mandioca e Fruticultura em recursos genéticos e melhoramento. In: QUEIRÓZ, M. A. de; GOEDERT, C. O.; RAMOS, S. R. R. (Eds.). Recursos genéticos e melhoramento de plantas para o nordeste brasileiro. Petrolina: Embrapa Semi-Árido; Brasília, DF: Embrapa Recursos Genéticos e Biotecnologia, 1999. Disponível em: <http://www.cpatsa.embrapa.br/catalogo/livrorg/ acerolabrasil.pdf $>$. Acesso em: 5 maio 2008.

OLIVEIRA, S. A. de. Análise foliar. In: SOUZA, D. M. G.; LOBATO, R. (Eds.). Cerrado: correção do solo e adubação. 2. ed. Brasília, DF: Embrapa Informação Tecnológica, 2004. p. 245-256.

VILLELA, D. M.; LACERDA, L. D. Dinâmica de elementos minerais em folhas de duas espécies arbóreas de cerrado. Revista Brasileira de Biologia, Rio de Janeiro, v. 52, n. 1, p. 151-160, 1992. 